\title{
NEW NAMES AND COMBINATIONS IN JAMESBRITTENIA (SCROPHULARIACEAE - MANULEAE)
}

\author{
O. M. HILLIARD
}

\begin{abstract}
A key is given to distinguish Jamesbrittenia, Camptoloma and Lyperia from Sutera, a genus with which they have hitherto usually been equated. Twenty one species and one subspecies of Jamesbrittenia are newly described, and 63 new combinations are made (two at varietal level).
\end{abstract}

As currently used, the name Sutera includes four genera: Camptoloma Benth., Jamesbrittenia O. Kuntze, Lyperia Benth. and Sutera Roth. The distinctions between the genera in their revised circumscriptions will be fully discussed in a revision of Manuleae now in preparation. In the interim, the following simple key will serve to distinguish them.

1a. Calyx with a distinct tube, one or both pairs of stamens exserted (included in 2 species), posticous filaments not decurrent down corolla tube, seeds amber-coloured, pallid or blue Sutera

1b. Calyx lobed nearly or quite to base (except in 1 species), stamens usually included (anticous pair shortly exserted in Camptoloma), posticous filaments decurrent, seeds black or shades of brown 2

2a. Leaf bases decurrent forming narrow wings or ridges down stem, seeds black, patterned with longitudinal rows of transversely elongated pits arranged like a chequer-board (easily seen with a hand lens or even the naked eye) Lyperia

2b. Leaf bases not decurrent, seeds shades of brown, testa reticulate (except in 2 species of Jamesbrittenia)

3a. Corolla tube cylindric, abruptly expanded near apex, stamens included (tips of anticous anthers rarely visible in throat), seeds with many longitudinal rows of cells forming a reticulum Jamesbrittenia

3b. Corolla tube cylindric, scarcely expanded at apex, anticous stamens very shortly exserted, seeds with 6-8 longitudinal ribs, reticulate between the ribs_Camptoloma

Jamesbrittenia accrescens (Hiern) Hilliard, comb. nov.

Basionym: Sutera accrescens Hiern in Thiselton-Dyer, Fl. Cap. 4(2): 305 (1904).

Jamesbrittenia acutiloba (Pilger) Hilliard, comb. nov.

Basionym: Lyperia acutiloba Pilger in Bot. Jahrb. 48: 440 (1912).

Jamesbrittenia adpressa (Dinter) Hilliard, comb. nov.

Basionym: Sutera adpressa Dinter in Feddes Repert. Beih. 53: 9 (1928).

Jamesbrittenia albanensis Hilliard species nova ab $J$. foliolosa (Benth) Hilliard foliis utrinque glanduloso-pubescentibus, pilis ad $0.15-0.3 \mathrm{~mm}$ longis (nec superne glabris vel 
fere glabris, subtus glandulis nitidis dense indutis, pilis glandulosis si adsentibus minutis); ab J. filicauli (Benth.) Hilliard floribus in racemos fere nudos (nec foliatos) dispositis et corollae limbo purpurascente (nec albo) differt.

Type: Cape, Albany div., Queen's Pass, $2000 \mathrm{ft}, 16$ viii 1964, Bayliss 2268 (holo. NBG, iso. $Z$ ).

Jamesbrittenia albiflora (Verdoorn) Hilliard, comb. nov.

Basionym: Sutera albiflora Verdoorn in Fl. Pl. S. Afr. pl. 485 (1933).

Jamesbrittenia albobadia Hilliard species nova a $J$. burkeana (Benth.) Hilliard et $J$. accrescente (Hiern) Hilliard corollae limbo texturae tenuis albo zona texturae crassae badia circum orem (nec texturae crassae, badio, margine pallida texturae tenuis) distinguenda. Type: Zimbabwe, W Bulawayo-Essexdale, Hope Fountain Mission, c.1400m, 20 vi 1974, Norrgrann 565 (holo. S).

Jamesbrittenia albomarginata Hilliard, species nova a J. argentea (L.f.) Hilliard foliis aut integris aut uno pare dentium prope apicem (nec in dimidio superiore grosse dentatis), floribus ad apices ramulorum tantum productis (nec per longitudinem caulis), corollae tubo $12-18 \mathrm{~mm}$ longo (nec 7-9.5 mm) et corollae lobis brunneis lateraliter albomarginatis (nec omnino albis) differt.

Type: Cape, Bredasdorp div., [c.3420], near Struys Bay, x 1940, Esterhuysen 5112 (holo. BOL, iso. NBG).

Jamesbrittenia amplexicaulis (Benth.) Hilliard, comb. nov.

Basionym: Lyperia amplexicaulis Benth. in Hook., Comp. Bot. Mag. 1: 377 (1836).

Jamesbrittenia angolensis Hilliard, species nova a $J$. carvalhoi (Engl.) Hilliard foliis facie superiore glandulis nitentibus solum indutis (nec pilis glandulosis c. $0.4-0.7 \mathrm{~mm}$ longis) et capsula etiam glandulis nitentibus solum induta (nec pilis minutis glandulosis) distinguitur.

Type: Angola, Huila, Sà da Bandeira, Tundavala, 24 vii 1962, Henriques 71 (holo. LISC; iso. $\mathrm{BM}, \mathrm{COI}, \mathrm{K})$.

Jamesbrittenia argentea (L.f.) Hilliard, comb. nov.

Basionym: Manulea argentea L.f., Suppl. 386 (1782).

Jamesbrittenia aridicola Hilliard species nova a $J$. megadenia Hilliard pilis glandulosis caulium et pedicellorum capitulis minus quam $0.1 \mathrm{~mm}$ diametro (nec pilis nonnullis dispersis capitulis $0.2-0.3 \mathrm{~mm}$ diam.), corollae lobis distincte emarginatis sinu $0.8-1.6 \mathrm{~mm}$ alto (nec integris vel leviter emarginatis sinu ad $0.3 \mathrm{~mm}$ alto) distinguitur. A $J$. thunbergii (G. Don) Hilliard corollae tubo 16-21.5mm (nec 7.5-17mm), corollae limbo albo (nec malvino), antheris alte in fauce insertis, apicibus antherarum anticarum ad summum $0.5 \mathrm{~mm}$ sub ore (nec antheris alte inclusis, apicibus antherarum anticarum c. $1.2-2.2 \mathrm{~mm}$ sub ore) recedit.

Type: Cape, Namaqualand, 2917DB, 20 miles NE of Springbok, 8 ix 1950, Maguire 340 (holo. NBG, iso. STE). 
Jamesbrittenia aspalathoides (Benth.) Hilliard, comb. nov.

Basionym: Lyperia aspalathoides Benth. in Hook., Comp. Bot. Mag. 1: 381 (1836).

Jamesbrittenia aspleniifolia Hilliard species nova ab $J$. aurantiaca (Burchell) Hilliard lamina foliorum ambitu oblonga (nec late elliptica nec ovata), semel vel bis fere ad costam secta (nec bis vel ter) lobis late ellipticis vel subrotundis, rotundatis (nec oblongis vel anguste ellipticis subacutis), utrinque glanduloso-pubescentibus, pilis $0.2-0.5 \mathrm{~mm}$ longis (nec pilis sparsis $0.1-0.2 \mathrm{~mm}$ longis pro maxime parte in pagina inferiore, pagina superiore glandulis nitidis fere omnino induta), corollae tubo breviore et latiore (4-5 x 3mm, nec 5-7 x 1.7-2mm), stylo 1.8-2.2mm (nec 3.2-4.5mm) longo differt.

Type: Cape, Barkly East div., 3028CC, Rhodes to Naude's Nek, Dunley, c.7500ft, 13 ii 1983, Hilliard \& Burtt 16615 (holo. E; iso. K, NU, PRE, S).

Jamesbrittenia atropurpurea (Benth.) Hilliard, comb. nov. Basionym: Lyperia atropurpurea Benth. in Hook., Comp. Bot. Mag. 1: 380 (1836).

Jamesbrittenia atropurpurea (Benth.) Hilliard subsp. pubescens Hilliard subsp. nov. a subsp. atropurpurea caulibus juvenilibus pedicellisque glanduloso-pubescentibus, pilis $0.15-0.5 \mathrm{~mm}$ longis differt.

Type: Botswana, $20^{\circ} 49.75^{\prime}$ S, $21^{\circ} 28.7^{\prime} \mathrm{E}, 18$ iii 1980, Smith 3247 (holo. SRGH).

Jamesbrittenia aurantiaca (Burchell) Hilliard, comb nov.

Basionym: Buchnera aurantiaca Burchell, Trav. S. Afr. 1: 270 (1822).

Jamesbrittenia barbata Hilliard, species nova a $J$. canescenti (Benth.) Hilliard ramificatione ramunculoidea (nec virgata) et corollae ore insigniter barbato, pilis saltem usque ad sinum labii postici attingentibus (nec pilis ad interiorem tubi fere restrictis in basin labii antici brevissime extensis) differt.

Type: Namibia, Swakopmund distr., 2214DB, Welwitschia Fläche bei Haigamkab, 1 v 1965, Giess 8738 (holo. WIND).

Jamesbritten beverlyana (Hilliard \& Burtt) Hilliard, comb. nov.

Basionym: Sutera beverlyana Hilliard \& Burtt in Notes RBG Edinb. 43: 216 (1986).

Jamesbrittenia bicolor (Dinter) Hilliard, comb. nov.

Basionym: Sutera bicolor Dinter in Feddes Repert. 30: 186 (1932).

Jamesbrittenia breviflora (Schltr.) Hilliard, comb. nov.

Basionym: Lyperia breviflora Schltr. in J. Bot. 34: 393 (1896).

Jamesbrittenia burkeana (Benth.) Hilliard, comb. nov.

Basionym: Lyperia burkeana Benth. in A. DC., Prodr. 10: 361 (1846).

Jamesbrittenia calciphila Hilliard, species nova a $J$. aspalathoide (Benth.) Hilliard pilis caulium et pedicellorum longioribus (c. $0.15-0.2 \mathrm{~mm}$, nec plerumque minus quam $0.1 \mathrm{~mm}$ longis) et foliis subrotundis vel late spatulatis (nec oblongis vel anguste spatulatis), longitudine plerumque 1-2.5: 1 (nec 2.5-7:1) distincta. 
Type: Cape, Riversdale div., 3421 BC, Canca se Laagte, 160m, 20 iii 1975, Oliver 5719 (holo. STE).

Jamesbrittenia candida Hilliard, species nova a $J$. grandiflora (Galpin) Hilliard pilis caulium et foliorum longis (ad 1-2mm) mollibus glandulosis (nec glanduloso-hispidis pilis ad $0.2-1 \mathrm{~mm}$ longis), glandulis nitentibus in caulibus foliis calycibus paucis (nec numerosis), inflorescentiis paucifloris, limbo corollae albo (nec malvino) distinguenda.

Type: Transvaal, Pietersburg distr., 2429 BA, Chunies Poort, 14 x 1938, Hafstrom \& Acock 1167 (holo. S, iso. PRE).

Jamesbrittenia canescens (Benth.) Hilliard, comb. nov.

Basionym: Lyperia canescens Benth. in Hook., Comp. Bot. Mag. 1: 379 (1836).

Jamesbrittenia canescens (Benth.) Hilliard var. laevior (Dinter) Hilliard, comb. nov. Basionym: Sutera canescens (Benth.) Hiern var. laevior Dinter in Feddes Repert. 30: 188 (1932).

Jamesbrittenia canescens (Benth.) Hilliard var. seineri (Pilger) Hilliard, comb. et stat. nov.

Basionym: Lyperia seineri Pilger in Bot. Jahrb. 48: 441 (1912).

Jamesbrittenia carvalhoi (Engl.) Hilliard, comb. nov.

Basionym: Cycnium carvalhoi Engl., Pflanzenw. Ost. - Afr. C 360 (1895).

Jamesbrittenia chenopodioides Hilliard species nova a $J$. canescente (Benth.) Hilliard habitu (caule principali e basi solitario, nec caulibus pluribus), indumento tantum e glandulis nitidis in caulibus, foliis, pedicellis, calycibus (pilis minutis glandulosis in marginibus loborum exceptis), ovariis, capsulis (nec pilis glandulosis $0.1-0.2 \mathrm{~mm}$ longis in pedicellis calycibusque adsentibus), corollae limbo plerumque longiore $(8-15 \mathrm{~mm}$, nec $7-9.5 \mathrm{~mm}$ ), corollae lobis luteis striis coloratis in basi quoque lobo absentibus nec lobis aut luteis aut rubris aut brunneis saepe stria mediana colore alio notatis differt.

Type: Namibia, Omaruru distr., 2114AB, Brandberg, Tsisab Valley, 20 ii 1963, Kers 1010 (holo. WIND).

Jamesbrittenia concinna (Hiern) Hilliard, comb. nov.

Basionym: Sutera concinna Hiern in Thiselton-Dyer, Fl. Cap. 4(2): 293 (1904).

Jamesbrittenia crassicaulis (Benth.) Hilliard, comb. nov.

Basionym: Lyperia crassicaulis Benth. in Hook., Comp. Bot. Mag. 1: 379 (1836).

Jamesbrittenia dentatisepala (Overkott) Hilliard, comb. nov.

Basionym: Sutera dentatisepala Overkott in Bothalia 7: 491 (1961).

Jamesbrittenia dolomitica Hilliard, species nova a J. heucherifolia (Diels) Hilliard pilis in partibus vegetativis crassioribus, petiolis plerumque brevioribus (ad $2.5-10 \mathrm{~mm}$ longis, nec 7-18mm), proportione lamina: petiolo 2.5-4.4: 1 (nec 1.1-2.4: 1) inflorescentiis magis congestis (internodio secudo basi inflorescentiae $2-8(-10) \mathrm{mm}$, nec (7-)10-16(-26)mm), pilis calycis $0.4-1 \mathrm{~mm}$ (nec $0.6-3 \mathrm{~mm}$ ) distinguitur. A $J$. acutiloba (Pilger) Hilliard foliis 
superne alternis (nec semper oppositis), pedicellis usque ad 6-18mm longis (nec $1-6 \mathrm{~mm}$ ), corollae tubo 12-14.5mm longo (nec 19-25mm) distincta.

Type: Namibia, Grootfontein distr., 1917 DB, near Meteor, 5000ft, 19 vii 1965, Leach \& Bayliss 13007 (holo. WIND, iso. S).

Jamesbrittenia elegantissima (Schinz) Hilliard, comb. nov.

Basionym: Lyperia elegantissima Schinz in Verh. Bot. Ver. Brandenb. 31: 192 (1890).

Jamesbrittenia filicaulis (Benth.) Hilliard, comb. nov.

Basionym: Lyperia filicaulis Benth. in Hook., Comp. Bot. Mag. 1: 380 (1836).

Jamesbrittenia fimbriata Hilliard, species nova a $J$. primuliflora (Thell.) Hilliard pilis c. $1 \mathrm{~mm}$ longis in basi labii antici et postice intra tubo decurrentibus (nec pilis c. $0.2-0.4 \mathrm{~mm}$ longis parte tubo superiore et antico fere restrictis), fauce corollae tantum flavo (nec colore flavo e tubo in bases loborum radiate) differt.

Type: Namibia, Lüderitz - Nord distr., 2415 DD, Vreemdelingspoort, 10 v 1976, Oliver, Müller \& Steenkamp 6528 (holo. PRE, iso. K).

Jamesbrittenia fleckii (Thell.) Hilliard, comb. nov.

Basionym: Chaenostoma fleckii Thell. in Vierteljahrsschr. Nat. Ges. Zürich 60: 408 (1915).

Jamesbrittenia fodina (Wild) Hilliard, comb. nov.

Basionym: Sutera fodina Wild in Kirkia 5: 79 (1965).

Jamesbrittenia foliolosa (Benth.) Hilliard, comb. nov.

Basionym: Lyperia foliolosa Benth. in Hook., Comp. Bot. Mag. 1: 380 (1836).

Jamesbrittenia fragilis (Pilger) Hilliard, comb. nov.

Basionym: Sutera fragilis Pilger in Bot. Jahrb. 48: 439 (1912).

Jamesbrittenia fruticosa (Benth.) Hilliard, comb. nov.

Basionym: Lyperia fruticosa Benth. in Hook., Comp. Bot. Mag. 1: 377 (1836).

Jamesbrittenia giessii Hilliard species nova a $J$. angolensi Hilliard foliis utrinque glanduloso-puberulis (nec glandulis nitentibus in pagina superiore tantum praeditis), corollae limbo supra glabro (nec glandulis nitentibus circum orem), capsulis glanduloso-puberulis glandulis nitentibus ad suturas restrictis (nec omnino glandulis nitentibus indutis, pilis absentibus) differt.

Type: Namibia, Otjiwarongo distr., 2116 AB, farm Otjihaenamaparero (OTJ 92), $17 \mathrm{v}$ 1978, Giess 15235 (holo. WIND, iso. M).

Jamesbrittenia glutinosa (Benth.) Hilliard, comb. nov.

Basionym: Lyperia glutinosa Benth. in Hook., Comp. Bot. Mag. 1: 378 (1836).

Jamesbrittenia grandiflora (Galpin) Hilliard, comb. nov.

Basionym: Lyperia grandiflora Galpin in Kew Bull. 1895: 151 (1895). 
Jamesbrittenia hereroensis (Engl.) Hilliard, comb. nov.

Basionym: Chaenostoma hereroense Engl. in Bot. Jahrb. 19: 150 (1894).

Jamesbrittenia heucherifolia (Diels) Hilliard, comb. nov.

Basionym: Chaenostoma heucherifolium Diels in Bot. Jahrb. 23: 475 (1897).

Jamesbrittenia huillana (Diels) Hilliard, comb. nov.

Basionym: Chaenostoma huillanum Diels in Bot. Jahrb. 23: 477 (1897).

Jamesbrittenia incisa (Thunb.) Hilliard, comb. nov.

Basionym: Erinus incisus Thunb., Prodr. 103 (1800).

Jamesbrittenia integerrima (Benth.) Hilliard, comb. nov.

Basionym: Lyperia integerrima Benth. in A. DC., Prodr. 10: 359 (1846).

Jamesbrittenia jurassica (Hilliard \& Burtt) Hilliard, comb. nov.

Basionym: Sutera jurassica Hilliard \& Burtt in Notes RBG Edinb. 40: 292 (1982).

Jamesbrittenia kraussiana (Bernh.) Hilliard, comb. nov.

Basionym: Chaenostoma kraussianum Bernh. apud Krauss in Flora 27: 835 (1844).

Jamesbrittenia lesutica Hilliard, species nova $J$. crassicauli (Benth.) Hilliard et $J$. pristisepalae (Hiern) Hilliard affinis ab ambabus floribus multo majoribus (corollae tubo 18-21 mm longo, nec 8-12mm) et corollae limbo albo (nec malvino vel luteo) statim distinguenda.

Type: Lesotho, 2929 AC, Phutha, 8000ft, 28 ii 1949, Compton 21604 (holo. NBG).

Jamesbrittenia lyperioides (Engl.) Hilliard, comb. nov.

Basionym: Chaenostoma lyperioides Engl. in Bot. Jahrb. 10: 253 (1888).

Jamesbrittenia macrantha (Codd) Hilliard, comb. nov.

Basionym: Sutera macrantha Codd in Fl. Pl. Afr. 30 t. 1162 (1954).

Jamesbrittenia major (Pilger) Hilliard, comb. nov.

Basionym: Lyperia major Pilger in Bot. Jahrb. 48: 441 (1912).

Jamesbrittenia maritima (Hiern) Hilliard, comb. nov.

Basionym: Sutera maritima Hiern in Thiselton-Dyer, Fl. Cap. 4(2): 277 (1904).

Jamesbrittenia maxii (Hiern) Hilliard, comb. nov.

Basionym: Sutera maxii Hiern in Thiselton-Dyer, Fl. Cap. 4(2): 288 (1904).

Jamesbrittenia megadenia Hilliard, species nova pilis glandulosis megacephalis in caulibus et pedicellis, capitulis $0.2-0.3 \mathrm{~mm}$ diam. (pilis similibus a speciebus omnibus affinibus absentibus) facile recognoscenda; etiam a $J$. aridicola Hilliard corollae lobis integris vel emarginatis sinu ad $0.3 \mathrm{~mm}$ alto (nec corollae lobis profunde emarginatis sinu c. $0.8-1.6 \mathrm{~mm}$ alto) distinguitur.

Type: Namibia, Bethanien distr., 2617 CC, farm Irene (BET 161), 8 vi 1976, Giess \& Müller 14278 (holo. WIND). 
Jamesbrittenia megaphylla Hilliard, species nova a $J$. bicolori (Dinter) Hilliard habitu herbaceo (nec fruticulo ramuloso), foliis majoribus (16-45 x 15-34mm, nec 8-27 x $3-16 \mathrm{~mm}$ ), pedicellis longioribus $(7-16 \mathrm{~mm}$, nec $2-6 \mathrm{~mm})$ recedit. A $J$. majore (Pilger) Hilliard foliis plerumque alternis (nec oppositis), latioribus in proportione longitudinis (1-1.3: 1, nec 1.3-2.6: 1), indumento caulis foliorum calycis breviore (pilis $0.5-1 \mathrm{~mm}$, nec $1-2.5 \mathrm{~mm})$, corollae tubo breviore $(9.5-10 \mathrm{~mm}$, nec $15-22 \mathrm{~mm})$, limbo minore (8-9mm trans lobos laterales, nec $13-20 \mathrm{~mm}$ ) albo (nec roseo nec violaceo) distinguenda.

Type: Namibia, Warmbad distr., 2817 DA, 12km W Nordufer, 7 viii 1976, Giess 14524 (holo. WIND; iso. M, PRE).

Jamesbrittenia merxmuelleri (Roessler) Hilliard, comb. nov.

Basionym: Sutera merxuelleri Roessler in Mitt. Bot. Staatss. München 16 (Beih.): 41 (1980).

Jamesbrittenia micrantha (Klotzsch) Hilliard, comb. nov.

Basionym: Lyperia micrantha Klotzsch in Peters, Reise Mossamb. Bot. 222 (1861).

Jamesbrittenia microphylla (L.f.) Hilliard, comb. nov.

Basionym: Manulea microphylla L.f., Suppl. 285 (1782).

Jamesbrittenia montana (Diels) Hilliard, comb. nov.

Basionym: Chaenostoma montanum Diels in Bot. Jahrb. 26: 121 (1899).

Jamesbrittenia multisecta Hilliard, species nova ab $J$. aurantiaca (Burchell) Hilliard foliis semper oppositis (nec sursum alternis), tenuius dissectis, lobis linearibus acutis (nec oblongis vel anguste ellipticis, subacutis), floribus ad axillas superiores restrictis (nec racemum longum formantibus), pedicellis brevioribus (ad summum 3-5mm, nec 7$25 \mathrm{~mm}$ ), calycis lobis dissectis (nec integris nec dente interdum ornatis) distinguenda.

Type: Transkei, Umtata - Engcobo road near Umtata, 21 xi 1977, Hilliard \& Burtt 10552 (holo. E).

Jamesbrittenia m yriantha Hilliard species nova ab J. dissecta (Del.) O. Kuntze inflorescentia paniculata (nec simpliciter racemosa), pedicellis $10-23 \mathrm{~mm}$ longis (nec 3-12 mm) et floribus majoribus (limbo $4-4.5 \mathrm{~mm}$ diam., nec $2-4 \mathrm{~mm}$ ), stylis $2-2.8 \mathrm{~mm}$ longis (nec $1.2-1.5 \mathrm{~mm}$ ) differt. Ab J. micrantha (Klotzsch) Hilliard habitu annuo (nec perenni), foliis profundius dissectis, corollae limbo albo (nec luteo), stylo $2-2.8 \mathrm{~mm}$ longo (nec 1.8 $2.2 \mathrm{~mm}$ ) distinguenda.

Type: Zimbabwe, Sebungwe distr., Simchembai Camp, 1500ft, ix 1955, Davies 1433 (holo. SRGH).

Jamesbrittenia namaquensis Hilliard, species nova a $J$. atropurpurea (Benth.) Hilliard foliis minute utrinque pubescentibus, subtus pilis et glandulis nitentibus intermixtis (nec glandulis nitentibus solum adsentibus), calyce pilis glandulosis stipitatis insuper glandulis nitentibus (nec glandulis nitentibus solum adsentibus) differt.

Type: Cape, Namaqualand, Richtersveld, 2817 AA, Kodaspiek, 2 ix 1977, Oliver, Tölken \& Venter 448 (holo. STE, iso. PRE). 
Jamesbrittenia pallida (Pilger) Hilliard, comb. nov.

Basionym: Lyperia pallida Pilger in Bot. Jahrb. 48 : 441 (1912).

Jamesbrittenia pedunculosa (Benth.) Hilliard, comb. nov.

Basionym: Chaenostoma pedunculosum Benth. in Hook., Comp. Bot. Mag. 1: 377 (1836).

Jamesbrittenia phlogiflora (Benth.) Hilliard, comb. nov.

Basionym: Lyperia phlogiflora Benth. in Hook., Comp. Bot. Mag. 1: 379 (1836).

Jamesbrittenia pilgeriana (Dinter) Hilliard, comb. nov.

Basionym: Lyperia pilgeriana Dinter in Feddes Repert. 19: 93 (1923).

Jamesbrittenia pinnatifida (L.f.) Hilliard, comb. nov.

Basionym: Manulea pinnatifida L.f., Suppl. 286 (1782).

Jamesbrittenia primuliflora (Thell.) Hilliard, comb. nov.

Basionym: Chaenostoma primuliflorum Thell. in Vierteljahrsschr. Nat. Ges. Zürich 60: 409 (1915).

Jamesbrittenia pristisepala (Hiern) Hilliard, comb. nov.

Basionym: Sutera pristisepala Hiern in Thiselton-Dyer, Fl. Cap. 4(2): 293 (1904).

Jamesbrittenia racemosa (Benth.) Hilliard, comb. nov.

Basionym: Lyperia racemosa Benth. in Hook., Comp. Bot. Mag. 1: 378 (1836).

Jamesbrittenia ramosissima (Hiern) Hilliard, comb. nov.

Basionym: Sutera ramosissima Hiern in Thiselton-Dyer, Fl. Cap. 4(2): 265 (1904).

Jamesbrittenia sessilifolia (Diels) Hilliard, comb. nov.

Basionym: Chaenostoma sessilifolium Diels in Bot. Jahrb. 23: 476 (1897).

Jamesbrittenia silenoides (Hilliard) Hilliard, comb. nov.

Basionym: Sutera silenoides Hilliard in Notes RBG Edinb. 43: 216 (1986).

Jamesbrittenia stellata Hilliard, species nova a $J$. aspalathoide (Benth.) Hilliard foliis ambitu late spatulatis (nec oblongis vel anguste spatulatis) et plerumque majoribus (2-7(27) $\times 0.8-3(-12) \mathrm{mm}$, nec $1.5-3 \times 0.3-1 \mathrm{~mm})$, corollae tubo longiore $(10-15 \mathrm{~mm}$ nec $9.5-11 \mathrm{~mm}$ ), corollae limbo stella lutea vel rubra circum orem notato distinquenda.

Type: Cape, Riversdale div., 3421 AD, Still Bay Hills, 9 viii 1949, Morris 257 (holo. NBG).

Jamesbrittenia stricta (Benth.) Hilliard, comb. nov.

Basionym: Lyperia stricta Benth. in A. DC., Prodr. 10: 360 (1846).

Jamesbrittenia tenella (Hiern) Hilliard, comb. nov.

Basionym: Sutera tenella Hiern in Thiselton-Dyer, Fl. Cap. 4(2): 278 (1904).

Jamesbrittenia tenuifolia (Bernh.) Hilliard, comb. nov.

Basionym: Lyperia tenuifolia Bernh. apud Krauss in Flora 27: 835 (1844). 
Jamesbrittenia thunbergii (G. Don) Hilliard, comb. nov.

Basionym: Manulea thunbergii G. Don, Gen. Syst. 4: 596 (1837-1838), precise date unknown).

Jamesbrittenia tortuosa (Benth.) Hilliard, comb. nov.

Basionym: Lyperia tortuosa Benth. in A. DC., Prodr. 10: 362 (1842).

Jamesbrittenia tysonii (Hiern) Hilliard, comb. nov.

Basionym: Sutera tysonii Hiern in Thiselton-Dyer, Fl. Cap. 4(2): 296 (1904).

Jamesbrittenia zambesica (R.E. Fries) Hilliard, comb. nov.

Basionym: Lyperia zambesica R.E. Fries, Wiss. Ergebn. Schwed. Rhod. Kongo Exped. 1911-1912, 1: 288 (1916).

Jamesbrittenia zuurbergensis Hilliard species nova a $J$. foliolosa (Benth.) Hilliard inflorescentiis foliatis, pedicellis plerumque minoribus (6-11 mm, nec $12-24 \mathrm{~mm}$ longis), corollae lobis apicibus rotundatis (nec truncatis nec retusis) pallide luteis (nec purpureis nec malvinis nec albis), ovario capsulaque omnino glandulis nitidis dense indutis (nec glandulis plus minusve ad suturas restrictis, alibi minute glanduloso-puberulis vel glabris) differt.

Type: Cape, Uitenhage div., 3325 AD, Rietberg, the mountain above Kirkwood, Zuurberg range, 10 vi 1962, Nordenstam 298 (holo. E, iso. S). 